\title{
DISTRIBUTED MODEL MANAGEMENT FOR COLLABORATIVE NETWORKS
}

IN AEC

\author{
Karsten Menzel \\ University College Cork, IRELAND, k.menzel@ucc.ie \\ Martin Keller
}

Dresden University of Technology, GERMANY, martin.keller@cib.bau.tu-dresden.de

\begin{abstract}
Projects in the building industry are extremely dynamic, driven by external conditions, modified user requirements and frequently changing business partners. Therefore, common principles should be established throughout the construction industry, which flexibly support the management of construction project information and processes. This paper presents an example of dynamic cross-enterprise process planning, execution and controlling on a conceptual and application level. It is based on a life-cycle oriented collaborative business process management model. The model supports collaborative business process modeling by using pre-defined process modules. The adaptation of the model to the specific requirements of the AEC\&FM-domain is illustrated by the example of errors and omissions management in Architecture, Engineering, and Construction (AEC).
\end{abstract}

\section{INTRODUCTION}

This paper discusses novel methods and tools to support inter-organizational collaborations within the AEC-industry more efficiently. Firstly, the concept of local and global knowledge in construction projects is introduced. Secondly, a potential model for the description of inter-organizational construction project management information is briefly explained. Important parts of this model include pre-defined process modules. Process modules document local (in-house) knowledge of an individual participant, which are then contributed to a collaborative network. Specific parameters have been identified to adapt process modules to the context of a certain project. The newly developed Process Module Chain modeling approach supports the integration of local process modules into a virtual, project-centered process chain, representing a complete collaboration network. As a third step, an integrated IT-architecture for the management and controlling of collaborative business processes is briefly introduced.

Last but not least, the authors will explain a mobile computing system for Errors and Omissions Management in AEC. This E\&O software system was developed to demonstrate the applicability of the methodological approach for distributed model management developed within the ArKoS research project (http://www.arkos.info). 


\section{APPROACH}

\subsection{Project Centric versus Organization Centric Approaches}

Construction projects are defined as complex one-of-a-kind projects. Thus, to derive a common model for collaborative construction project management, its complexity has to be reduced by subdividing it into integral/coherent sub-projects or project views. Therefore, the entire project has to be decomposed into its controlling elements and structured in a reasonable manner.

A requirement within collaborative networks is to clearly distinguish between publicly available "global" knowledge and specific technological knowledge, including business secrets of individual collaboration partners (so-called "local" knowledge). The Architecture for Integrated Information Systems (ARIS) (Scheer, 1999) supports the definition of different views in business process models. Global knowledge is represented on a vertical axis, including the organizational view and the output view, which are necessary to establish a goal-oriented collaboration. Local knowledge is represented on a horizontal axis. Local knowledge is bilaterally shared between partners (Zang et al., 2004).

Following the approach of global and local knowledge, an AEC-specific Construction Network Schema was developed (Keller et al. 2004\&2005) to specify the global knowledge in a more detailed way. The Construction Network Schema consists of four major elements: Project Organization, Project Structure, Project Information and Project Phase. The dimension, Project Organization, is subdivided into the categories, Organizational Structures and Roles, characterizing the management aspects of a project (global knowledge). The decomposition of the project into technical and functional aspects is realized by means of the dimension, Project Structure. High-level tasks -necessary for the completion of a project- are defined by the category, Function; whereas, the category, Building Object, structures the project into spatial and/or physical sections. The dimension, Project Information, defines the IT-Infrastructure and systems for inter-enterprise information exchange. It also specifies the Information Content that is exchanged amongst project partners.

\subsection{The Concept of Business Process Modules}

Business Process Modules (BPM) store and preserve local knowledge in a reusable and coherent manner. The aim of introducing Business Process Modules is to support the integrated use of local and global knowledge within a project team in a secure way. Process modules are generally pre-defined for the performance of a certain bundle of activities and are adaptable to different project contexts. Each process module represents a logical element with distinct interfaces (Menzel, 2003). These process interfaces are developed for a seamless integration of instantiated process modules into the existing Process Module Chain, defining all relevant input and output parameters. Each process module will be identified by certain metainformation that describes the parameters needed for its selection, initialization and integration. A schematic representation of a process module for construction processes is given in Figure 3. The generic processes (modeled as an Event-driven Process Chain) will be identified by Context and Initialization Parameters as well as Input and Output Information. 


\section{COLLABORATIVE NETWORK INFORMATION TO SUPPORT ERRORS \& OMISSIONS MANAGEMENT (E\&O)}

Currently, E\&O management, in particular E\&O documentation and inspection on the site, is little supported by software applications. Additionally, different inhomogeneous IT-formats are common practice. Therefore, it was decided to prototypically develop and implement an E\&O management system, running on mobile wireless devices (such as PDA). The system architecture should support easy integration into already existing, desktop-based IT environments.

E\&O management involves numerous organizations of different size and varying roles. It also comprises main as well as supporting functions. Furthermore, a precise specification of the building structure is required for E\&O management.

The development of the showcase started with a project analysis, which led to a general specification of typical constraints and requirements for E\&O management. Building sites of heterogeneous structure and different organizational types were examined. On the basis of the BPM developed, we demonstrated that seamless information exchange between different project participants can be organized more efficiently; especially, that existing global and local knowledge could be used as part of the mobile application.

To model global knowledge for E\&O management, the following definition is introduced (cf. Figure I): E\&O management processes belong to the "ConstructionPhase" of a building project. A common Organizational Structure in this phase is the "General Contractor" model. This model is composed of three major partners: a) the client, b) the general contractor, and c) subcontractors. The client contracts the general contractor for the installation of the complete building or major parts of it. The general contractor might be either one single company or a consortium of two or more companies. In each case, the general contractor usually assigns several subcontractors to distinct tasks. Each of these organizations has its own internal structure. However, for E\&O-management, all companies have to establish the Role "Quality Manager".

Each project is characterized by its individual Building Objects as output of the network. The availability and accessibility of that information is required if a socalled "context-sensitive" application is to be configured. In our showcase, additional SOAP-services were implemented supporting the information transfer from desktop-based project management applications to the mobile E\&O management system. In this way, the existing global knowledge of the project can easily become an integrated part of the mobile E\&O-system. The global project workflow was modeled by using the Process Module Chain approach, which is part of the ARIS-methodology. 


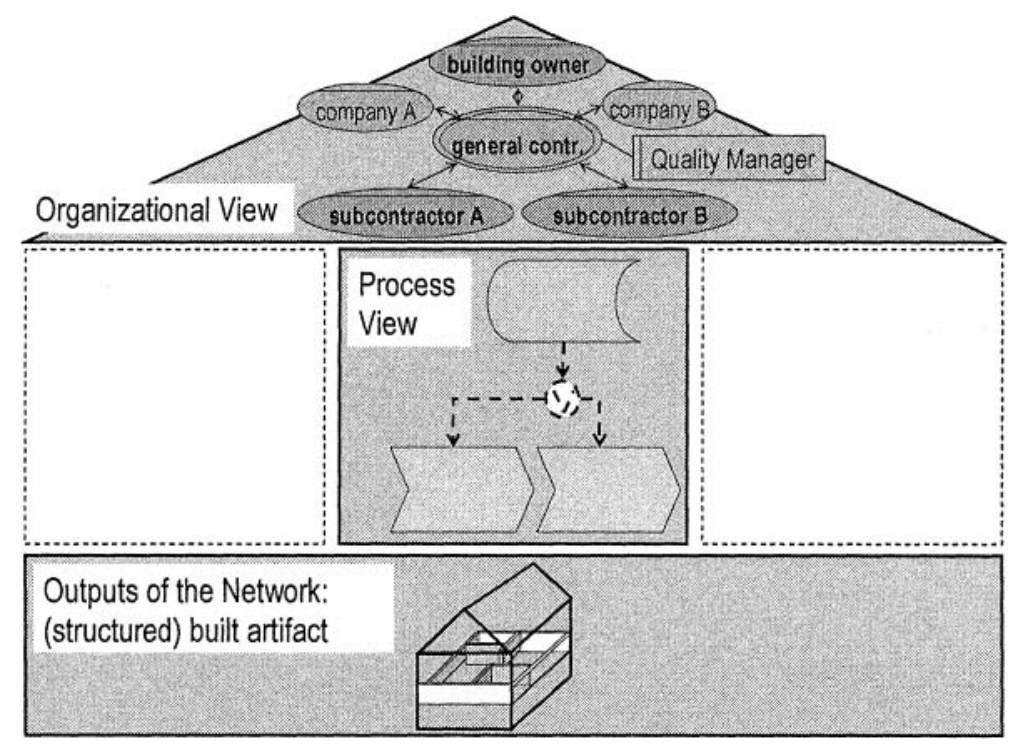

Figure 1 - Global knowledge of E\&O management

However, additional local knowledge must complement global knowledge, describing the individual steps of how to fix detected errors and omissions appropriately. Therefore, the Project Information Structure indicated on the left of Figure 2 has been developed and implemented. The developed service-oriented architecture allows mobile and office applications to access two servers: one storing the E\&O documentation and the other managing all project-related information, such as roles, participants, building product specifications, etc.

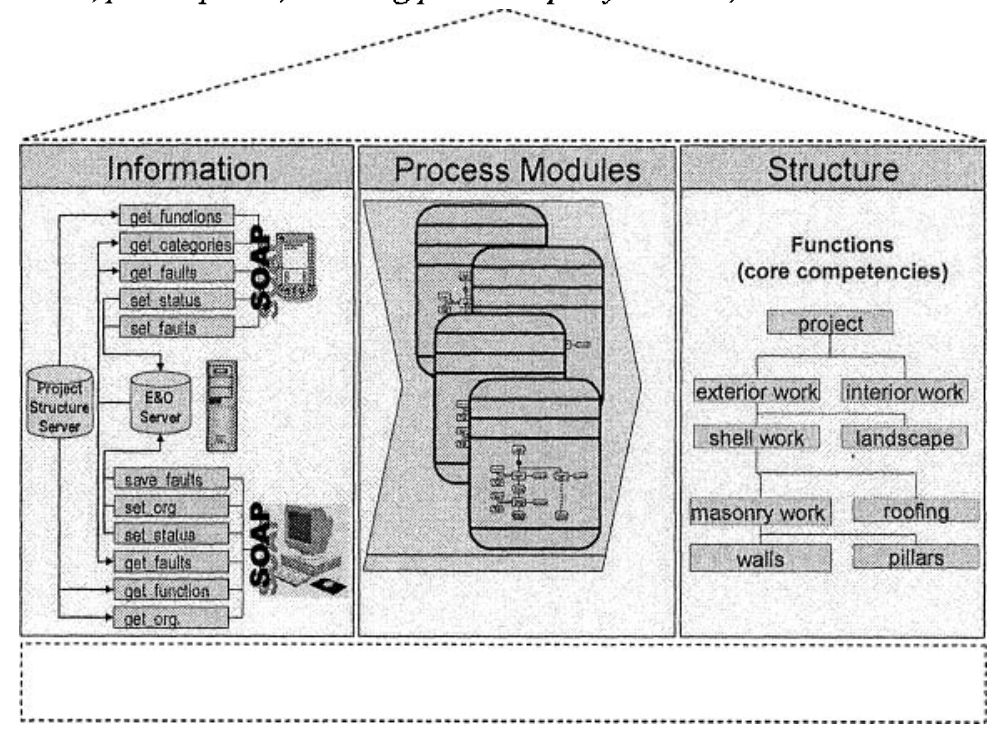

Figure 2 - Local knowledge of $\mathrm{E} \& \mathrm{O}$ management 
The communication between the various applications and different servers is realized by web services. Information exchange is handled by XML based SOAP messages. The data structure for the exchange of E\&O information and E\&O categories was developed and is exchanged by WSDL-specifications. The usage of such an open, transparent, service-oriented architecture supports the integration of existing applications. Thus, each partner can participate in E\&O management processes by using his own application(s) complemented by the mobile application.

This approach protects, on the one hand, the consistency of global collaborative network information and ensures, on the other hand, the extension of the projectspecific knowledge space by homogeneously adding local knowledge.

\subsection{Reference process models to support E\&O management}

At present, no standardized model for $\mathrm{E} \& \mathrm{O}$ management processes has been realized. Regulations are handled in a project-specific way. Therefore, we developed a general process model for E\&O-management, synthesizing the findings of our intensive project studies. Subsequently, this detailed model was decomposed into coherent process modules based on the modeling methods introduced in chapter 2 . Thus, the developed process modules can be applied to different construction project types. The various process modules are stored in the Repository of the Architecture for Collaborative Scenarios (Theling et al. 2005).

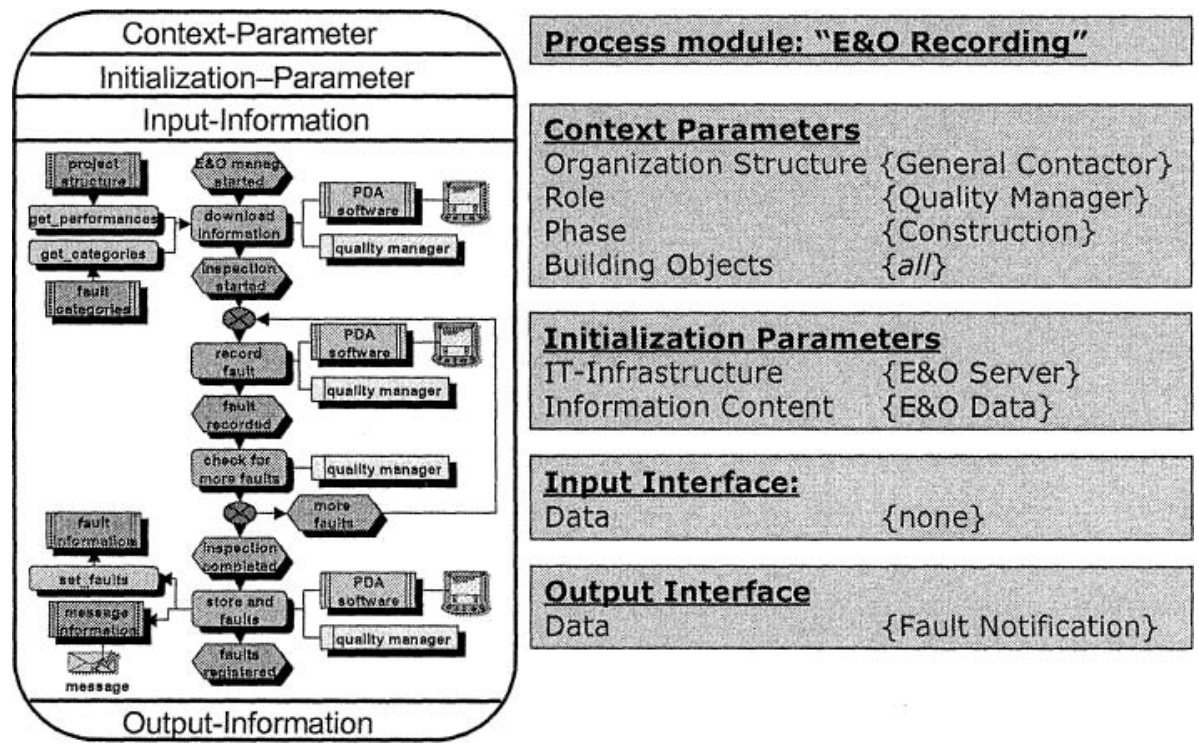

Figure 3 - Process module "E\&O Recording" (Event-driven Process Chain)

For the identified process modules, the meta-information described in chapter 2.3 has been specified. An example of the "E\&O Recording" process module is given in Figure 3. This module can be applied by the quality manager of the general contractor and is applicable for all major types of building objects. For the 
initialization of the process module, a server for $\mathrm{E} \& \mathrm{O}$ data management has to be established in the project. No input-data is required to start the process module. At the end of the process, a "fault notification" will be sent to the responsible partner.

\section{APPLYING THE ARCHITECTURE}

A general system architecture model developed in the ArKoS-project (Theling et al. 2005) was adjusted to our showcase as depicted in Figure 4. Firstly, a set of E\&O reference processes was developed which supports the modelling of cross-company collaborative networks. Secondly, complementary project-specific process descriptions were made accessible by using the SOAP-services described above. The E\&O-process modules and the project specific functions are both part of the process-data-pool.

Thirdly, diverse AEC-modeling tools, such as CAD-tools and their inherent standards (e.g. IFC, STEP AP 225), can be used to produce a complete model of the built artifact. The technical and structural information of the building are both combined in the project-data-pool. In addition, project documents can be attached to the process and project data by a document management application.

Furthermore, existing E\&O-protocols were analyzed in depth in order to define a comprehensive but general catalogue of $E \& O$ descriptions. These descriptions were categorized, leading to a complete E\&O classification.

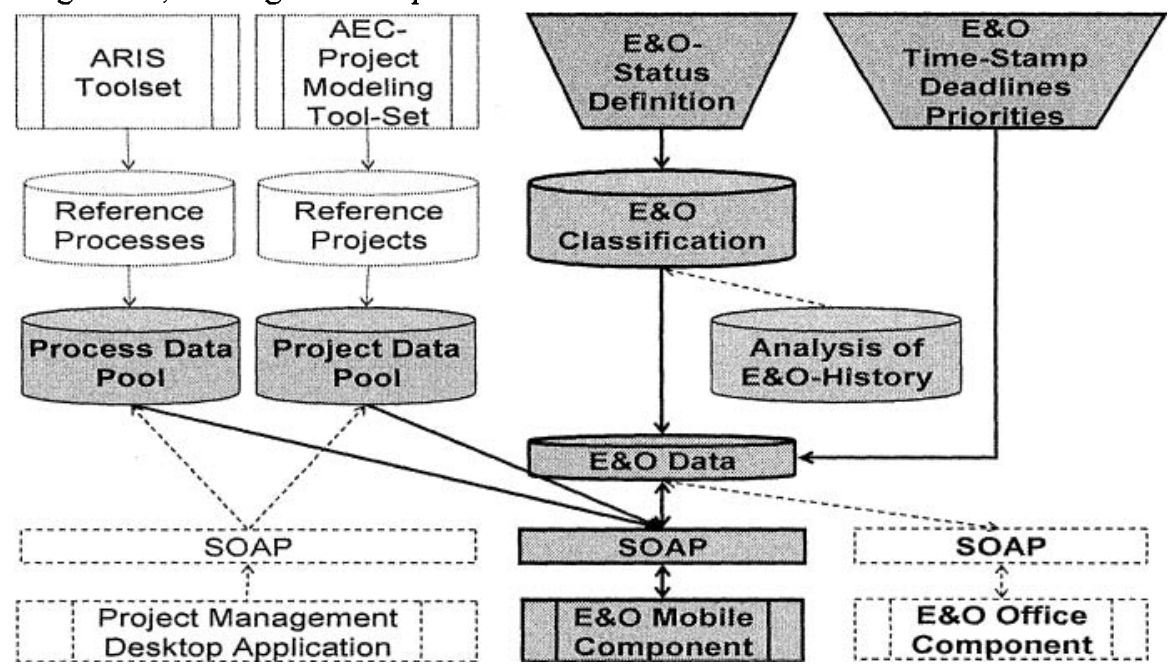

Figure 4 - System Architecture to support „Errors and Omissions Management”

The process-data-pool, project-data-pool and E\&O classification are part of the global E\&O-knowledge base, supporting a specific collaborative E\&O-network. This knowledge-base delivers the input data for the mobile $\mathrm{E} \& \mathrm{O}$ system component. The accessibility of these knowledge components reduces data acquisition efforts, contributes to consistent data management and supports an effective, holistic project management for all participants of the $\mathrm{E} \& \mathrm{O}$ collaborative network.

The developed E\&O system architecture also contains two management components. During build-time, nine different states were defined, describing the 
"life-cycle" of an error or omission. The precise definition of the different states allows at each time of the project the evaluation and analysis of the work progress with regard to E\&O-management. The following states were defined: (1) E\&O registered, (2) in progress by general contractor, (3) in progress by subcontractor, (4) rejected by subcontractor, (5) under negotiation with subcontractor, (6) completed by subcontractor, (7) completed by general contractor, (8) rejected by general contractor, (9) completed, (10) under negotiation with client.

Finally, for each error or omission a time-stamp is generated. Additionally, the user can define a priority and deadline for each detected error or omission. This data is generated during "build-time" and allows exact controlling of E\&O management processes.

\subsection{Errors and Omissions application}

The mobile E\&O-system component consists of three parts: (1) data acquisition, (2) data analysis, and (3) synchronization. Within this section, the first two parts are described in more detail.

The GUIs depicted in Figure 5 illustrate the data acquisition sequence supported by the mobile system component. On the first screen, the user must localize the error. Most of the information is delivered from the project-data-pool (e.g. building, floor, room). The user only selects this information instead of needing to acquire this information redundantly. The second screen supports the error or omission specification. Again, the necessary information specifying the profession and the building part is delivered by the process-data-pool. The error category as well as a pre-defined error description is selected from the error catalogue.

Finally, the application automatically adds the current date and deadline. The user might wish to specify the cost necessary to fix the error in addition to specifying the original company responsible for fixing the error. The information of the responsible company (subcontractor) is already specified in the project-data-pool and can be selected according to the specified profession (e.g. electrician, plumber, painter, etc.).
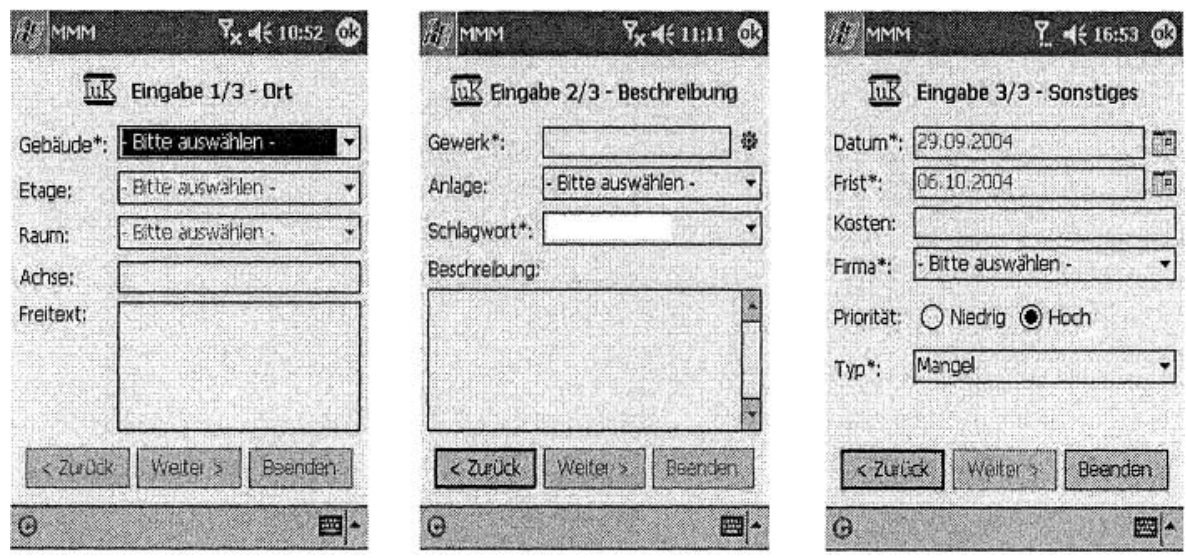

Figure 5 - Screen-shot Mobile Application for Error and Omission Management (Ort=Localization, Beschreibung =Specification, Sonstiges =Costs \& Deadlines) 
The detailed and precise error \& omission specifications are stored temporarily in a local database on the mobile device. The status of all new acquired errors is "1=registered". The local and the main "E\&O-database" are synchronized by analyzing the E\&O-states and using the SOAP-services, as described above.

All errors and omissions will be propagated without delay to the responsible partners of the collaborative network. The partners can act quickly and immediately to fix an error or omission. Short response times contribute to decreased costs of errors \& omissions management and to a higher quality of the built artifact. Finally, the client is served in a better way, and the overall costs for delivering the built artifact are decreased.

\section{CONCLUSION}

A showcase, based on the example of errors and omissions management in Architecture, Engineering, and Construction, was developed and implemented. To achieve that, the required global and local knowledge was identified, and a general business process model was developed and decomposed into coherent process modules. Additionally, a mobile application was implemented to demonstrate the basic functionalities of the proposed system architecture. The application was successfully tested in demonstration projects of various sizes ( $€ 5 \mathrm{Mill}$. to $€ 50 \mathrm{Mill}$.).

The work has been conducted within the scope of the project 'Architecture for Collaborative Systems' (ArKoS - www.arkos.info) funded by the German Ministry for Education \& Research since 2003. The design, realization and analysis of the E\&O management showcase were supported by the Bilfinger und Berger AG. The mobile component will become a part of the company's software system in 2006 .

\section{REFERENCES}

1. Keller M., Katranuschkov P. and Menzel K. (2004). Modeling collaborative processes for Virtual Organizations in the building industry, In proceedings of ECPPM, Balkema.

2. Keller M., Menzel K. and Scherer R.J. (2005). Towards a Meta-Model for Collaborative Construction Project Management. In proceedings of PRO-VE 05, Valencia, Spain.

3. Menzel K. (2003). Nachhaltiges Ressourcenmanagement mit mehrdimensionalen Informationssystemen. Habilitation (post-doctoral lecturer thesis), Dresden University of Technology, Germany.

4. Scheer A.-W. (1999). ARIS, Business Process Frameworks, Berlin et al., Springer.

5. Theling, T., Zwicker, J., Loos, P., Adam, O., Hofer, A.; „Enabling Dynamic Networks Uing an Architecture for Collaborative Scenarios"; in: Proceedings of the $22^{\text {nd }}$ Conference on Information Technology in Construction (CIB-W78); TU Dresden, Germany (ISBN 3-86 005-478-3).

6. Zang S., Hofer A and Adam O. (2004). Cross-Enterprise Business Process Management ArchitectureMethods and Tools for Flexible Collaboration, In R. Meersman, Z. Tari \& A. Corsaro (eds.) Proc. On the Move to Meaningful Internet Systems 2004: OTM Confederated International Workshops and Posters, GADA, JTRES, MIOS, WORM, WOSE, PhDS, and INTEROP 2004, October 25-29, Agia Napa, Cyprus. 